\title{
Epstein-Barr Virus and its Capacity to Evade the Immune System to Cause Cancer
}

\section{Anirban Goutam Mukherjee ${ }^{1}$, Uddesh Ramesh Wanjari ${ }^{2 *}$, Fereshteh Ramezani $^{3}$, Aishwarya Laxmi ${ }^{4}$, Piyush Jagdish Balgote ${ }^{5}$ and Surbhi Balwant Dhoke ${ }^{2}$}

${ }^{1}$ Department of Biosciences, School of BioSciences and Technology, Vellore Institute of Technology (VIT), Vellore, Tamil Nadu, 632014, India

${ }^{2}$ Department of Biochemistry, Kamla Nehru Mahavidyalaya, Nagpur, Maharashtra, India

${ }^{3}$ Student Research Committee, School of Medicine, Islamic Azad University, Mashhad

Branch, Mashhad, Iran

${ }^{4}$ Department of Biotechnology, Institute of science, Banaras Hindu University, Varanasi, Uttar Pradesh, India

${ }^{5}$ Molecular Biology and Genetic Engineering, RTM Nagpur University, Nagpur,

Maharashtra, India

*Corresponding Author: Uddesh Ramesh Wanjari, Department of Biochemistry,

Kamla Nehru Mahavidyalaya, Nagpur, Maharashtra, India.
Received: July 10, 2021

Published: August 07, 2021

(C) All rights are reserved by Uddesh

Ramesh Wanjari., et al.

\begin{abstract}
Epstein-Barr virus (EBV) is a commonly known herpes virus that contains tegument, envelope, DNA, and a core with an icosahedral nucleocapsid. Malignant EBV has been implicated in developing Burkitt's lymphoma, lymphoma, nasopharyngeal cancer, Hodgkin's disease, and non-Hodgkin's lymphoma. Gene rearrangements in the immunoglobulin variable region of lymphoma neoplastic cells indicate their origin B-cells in the germinal center. EBV can infect B-cells at all stages and express growth genes. This review focuses on an in-depth idea of how the virus escapes the immune system to cause cancer. Additionally, this work also sheds light on the association of the EVB in various types of cancers like Burkitt's lymphoma, Non-Hodgkin's Lymphoma, and Leiomyosarcomas.
\end{abstract}

Keywords: Epstein-Barr Virus (EBV); CD 56; miRNA; CDKs; T-cell

\section{Introduction}

\section{Characteristics of EBV in cancer}

Interestingly, the only host for EBV is human beings. The primary EBV infection involves saliva transfer into the stratified squamous epithelium of the oropharynx. The virus possibly enters via the tonsils, where the primary lytic, latent virus infection, and Blymphocytes, can be accessed [1].

In the initial infection, the virus spreads from the oropharynx to the saliva for several years; after the virus has colonized the
B-lymphoid compartment, latency can reactivate in any mucosal membrane where B-cells exist [2].

The same patterns of latent protein production initially indicate that virus-induced B-cell variation can directly increase the effectiveness of genes in lymphoma types and lymph proliferative abnormalities, and in B-memory cell lines (called phenotypes): Class I, Class II, and latency Class III (for development, rebound, and latency of gene expression programs) [2]. The EBV genome is retained in these cells by incorporating the viral genome into the host chromosome, either a cyclic multicopy episode or a single copy. The 
EBV genome is stored inside the cell without killing it and triggering its immune system. Finally, the virus activates cell growth regulation mechanisms $[1,3,44]$.

The cells are inhibited in such a way that only the EBNA-1 protein is expressed. Although cytotoxic reactions to EBNA-1 are rare, its expression is essential in lymphocytes that evade immune control [4].

\section{Association with malignancy}

EBV has many latent genes, especially those associated with immunodeficiency diseases such as LMP1 and 2A (Latent Membrane Protein-1 and 2A), and the expression increases the proliferation of EBV-infected plastic B-cells. EBNA3C spreads mutations by quickly passing checkpoints during cell division and causing malignant cancers; in contrast, the natural cycle of infected cells generates naive blast B-cells. Non-native B-cells prevent changes in the cell's latency phase, causing the cell to remain irreversible in the growth phase, leading to AIDS-associated lymphomas and PTLDs $[2,5]$.

\section{Burkitt's lymphoma}

Burkitt's lymphoma is an invasive lymphoma in which the c-myc oncogene (present on chromosome 8) is moved to the heavy chain of chromosome 14 or the light chain of chromosome 2 or 22 , resulting in uncontrolled expression of the c-myc oncogene [8].

There are two types of Burkitt's lymphoma: endemic with EBV and non-endemic without EBV, where the breakpoints of the gene and its connection are different. The first type is known to affect 90\% of black people in Africa and Papua New Guinea with Akata Burkitt's lymphoma cell line [6]. The presence of latency-I gene Bcell stimulation is noted due to constant malaria reinfection. EBV influences the Fas-mediated apoptotic mechanism, which results from the regulation of caspase- 8 and the FLICE-inhibiting protein. In addition, EBV is responsible for enhancing and maintaining the anti-apoptotic protein of BCL-2 lymphocyte lines [7].

Continuous transformations of the immunoglobulin gene imply that B-Lymphocyte (BL) cells are generated from EBV-associated germinal center B-cells. All types of BL have variants in the c-Myc gene, leading to a dysregulated expression of c-Myc and promoting neoplastic cells' growth. The continuous exchange of $V$ (D) J strongly suggests that EBV is present in all cells. A lack of EBNA2 activity in BL cells is believed to be a key event in cancer. BL cell formation could be viewed as a risk factor and undermines the cellular machinery that usually protects against such changes and enables the spread of oncogenic processes. The presence of latent EBV in EBV-positive BL isolated neoplastic cells implies that EBV is necessary for BL cell viability. The role of EBV in the production of EBER is based on investigated tumor plasmids. EBNA1 and EBERs can promote the malignant development of BL cells in the absence of any other EBV inactive products; they also increase resistance to apoptosis from cycloheximide, glucocorticoids, and hypoxia [5,40]. EBER1 can play a role in growth factor control and cytokine gene regulation, as a pro-proliferative cytokine involved in the viability and transformation of B-cells and higher concentrations in EBV positive BL tumor cells [8].

\section{Non-hodgkin's lymphoma}

T/NK cell lymphoma and angioimmunoblastic lymphadenopathy include two types of direct EBV-associated lymphomas without affecting B-cells. T/NK cell lymphoma typically occurs in the nose and upper areas and has several related genotypic and phenotypic characteristics, including a lack of T-cell antigen and receptor gene recombination and natural killer cell marker activation CD 56. A colony of B cells characterizes angioimmunoblastic lymphadenopathy along the T-cells side [9].

\section{Leiomyosarcomas}

Lemiosarcomas are the tumors in the smooth muscle that causes the pathogenesis of leiomyoma and leiomyosarcoma in people with immunodeficiency such as AIDS. Some studies support the hypothesis that EBV may be involved in developing immunocompromised patients' smooth muscle tumors, but some believe that the fusion of healthy non-lymphoid cells with infected cells is the key to the virus entering these cells [10].

Smooth muscle infection with EBV or malignancy causes leiomyosarcoma. In addition to smooth muscle, the EBV receptor CD21 is also found in striated muscle and other cells. The virus is also found in the plasma of infected immunosuppressive individuals to a significant extent, increasing its receptor and reducing the immune system's strength, which plays a role in causing smooth muscle tumors [11].

\section{Induction of signaling pathways for survival}

LMP1 (Latent Membrane Protein-1) and LMP2A (Latent Membrane Protein-2A) are EBV proteins that play an essential role in cancer progression [5]. The latent membrane protein-1 can control 
the invasion and metastasis of epithelial cells, causing the production of IL- 6 and IL-8 and downregulate E-cadherin, matrix metalloproteinase (MMP), and sensitive epidermal growth factor (EGFR) [12].

The expression of LMP1 is rarely seen in EBV, but LMP2A protein can show a frequency of 40 to $50 \%$. It also stimulates cell growth, increases cell movement, attachment, and also downregulate cellular differences, which may be an epithelial cell process shown to activate signals in epithelial cells, including NF-kB, phosphoinositide-3-kinase (PI3K)/Akt, and signal transduction activator (STAT), which is an important treatment goal that can be achieved $[8,12]$.

Cyclooxygenase-2 (COX-2) is a persuadable cyclooxygenase isoenzyme that causes prostaglandin E2 (PGE2) to develop in response to variations in inflammatory stimuli or growth factors. CTAR1, CTAR2, both functional domains of LMP1, can cause COX-2 transcription, which stimulates the passage of the NF-kB signal to promote NPC development $[5,12]$.

The MAPK signaling pathway plays a vital role in regulating COX-2 expression in a large number of tumors. The activity of the COX-2 promoter alone regulates gene expression, protein levels, or PGE2 secretion. EBV has various mechanisms to regulate COX2; For example, COX2 is expressed in NPC samples while being associated with lymph node by LMP1 and metastasis by NFKB; or a reduction in COX2 mRNA expression and PGE2 secretion [12].

There are conflicting results from LMP1 overexpression. Some claim that LMP1 classically activates NFKB in kidney cells by using TRAF1 and TRAF2, but other recent studies suggest that overexpression of LMP1 and LMP2A can have a significant inhibitory effect on TRAF2 through sustained or transient transfection. Therefore, a viral mechanism for the continuous activation of NFKB can reduce the regulation of TRAF2 [5].

LMP1 and LMP2A regulate the COX2 inhibitory pathways through TRAF2 inhibition by MAPK or JAK-STAT pathways. The high expression of LMP1 reduces the expression of COX2 and also reduces pERK [12]. By inhibiting COX-2 inhibition, proliferation decreases and apoptosis increases, which may be due to the downregulation of the anti-apoptotic gene Bcl-2 and an increase in the apoptotic gene-Bax. COX2 can also improve angiogenesis through the expression of Akt and reduce the effect of E-cadherin on cancer invasion by increasing metastasis $[5,12]$.
Relation of microRNA with virus-induced cancers

Immune evade

EBV expressed miRNA is responsible for altering the immune function of the host and promoting cell survival. miRNA BART1 has been found to inhibit different components of the TGF- $\beta$ signaling pathway: an important cytokine regulating host immune response on infection with EBV and miRNAs such as BART 12, 4, 5, 18, 22 inducing some cytokine which prevent immune system activation. Some other miRNAs, such as BART16, block IFN- $\alpha$ mediated antiproliferative function in BL-cell by reducing the expression of CREB binding protein (CREBBP) [15]. Also, BHRF1-2-5p affect IL1receptor1(IL-1R), which prevent IL1 activation, required for innate immune activation, causing inhibition of cytokine expression in EBV infected cell. miRNA also causes inactivation or reduced expression of IL-receptors and IL, respectively; BART-6-3p was found to inhibit IL-6R causing lymphomagenesis, and BART3 reduced IL-6 expression by affecting importin-7 (IPO7). These showed that miRNA causes inhibition of different cytokine signaling in host cells [13].

Some miRNAs regulate the host cell's innate immunity; BART6$3 p$ inhibits the RIG-I signaling receptor, which acts as a pattern recognition receptor (PPR); it inhibits cell infection and the type I interferon (IFN). miRNA, BART20-5p, and BART8 affect IFN- $\boldsymbol{\gamma}$ and STAT1 pathway that causes TP53 suppression and nasal NK cell lymphoma progression [14]. BART2-5p helps to escape the virus from immune cell recognition and prevent cell killing by inhibiting MICB; it functions by increasing its expression on the cell surface in virus-infected cells. BART5-5p induces viral latency and NPC progression by activating specific pathways. miRNA takes part in inhibition of T-cell response, causing a halt in immune cell scrutiny; also preventing CD4+ cell action in the infected viral cell. BART1, 2, BHRF1-2 downregulates IL-12 expression in the infected cells, leading to inhibition of T-helper type1 (Th-1) cells and inhibiting some lysozyme enzymes such as cathepsin-B, and IFN- $\boldsymbol{\gamma}$ inducible protein 30 (IFI30) [13-15].

Most of miRNA such as BHRF1-13, BHRT-17, 3,16 are involved in inhibiting MHC-II mediated antigen processing by aborting some of the receptors that mediate processing of antigen such as TAP2, CD4+ cell, CD8+ cells, while BART-22 causes cells to pass off immune scrutiny and promote tumorgenesis by affecting LMP2A [13].

Citation: Mukherjee, A.G., Wanjari, U.R., Ramezani, F., Laxmi, A., Balgote, P.J., and Dhoke, S.B. "Epstein-Barr Virus and its Capacity to Evade the Immune System to Cause Cancer". Acta Scientific Microbiology 4.9 (2021): 52-62. 
In EBV-related GC overexpressed PD-L1, when IFN- $\boldsymbol{\gamma}$ was secreted from T-cell, it escapes immune response with the help of the PD1/PD-L1 pathway. The high level of expression of PD1/PD-L1 is associated with abnormalities in the PD-L1 3' UTR region. PD-L1 causes cell cycle arrest of T-lymphocytes, while anti-PD-L1 antibodies such as nivolumab when administered to gastric cancer patients, showed a better survival rate and were influential in treating recurrent cancer $[8,9]$. This antibody prevents cell cycle arrest of T-cells, causing an immune response towards EBV. In miRNA570, two single nucleotide polymorphism (SNP) causes over-amplification of the gene that codes for PD-L1 and JAK2 were seen in all GC caused by EBV [15].

\section{Control of cell growth}

EBV has been associated with malignancy and carcinogenesis. EBV miRNA is transcribed from two parts: BaHI-A region rightward transcript (BART) and BamHI fragment $\mathrm{H}$ open reading frame I (BHRF1) - cluster shown to inhibit different pathways that lead to cancer inhibition. Studies have shown that one of the miRNAs called BART7, over-activating TGF signaling, is involved in NPC's cancer progression $[8,17,41]$. BART transcript upregulation interferes with a pathway involved in cell proliferation, apoptosis, invasion, and metastasis, such as activation of p53, transforming growth factor- $\boldsymbol{\beta}$ and wnt signaling pathway that causes tumor progression. BART10 causes downregulation of $\boldsymbol{\beta}$-transducin repeats containing E3 ubiquitin-protein ligase $(\boldsymbol{\beta}$-TrCP), which prevent the destruction of $\boldsymbol{\beta}$ - catenin [17]. Overexpression of BART7-3p is found in NPC promoting tumor growth, even BART19-3p shown to reduce the expression of wnt inhibitory factor 1 (WIF1) and two of the tumor suppressor pathways [nemo like kinase (NIK), adenomatous polyposis coli (APC)] causing proliferation of GC cells. BART6$3 p$ and miR-14 cause down expression of IL-6R and PTEN, causing EBV infected BL cells to proliferate. BHRF1,2 is involved in reducing the expression of PRDMI - a tumor suppressor gene causing cell proliferation and apoptosis of LCL cells [18].

miRNA plays a crucial role in tumor cell proliferation and survival. BART4-5p has been shown to inhibit BH3 interacting domain death antagonist (BID) - a pro-apoptotic protein resulting in reduced GC cell apoptosis; BART-16 causes inhibition of BAX-associated apoptosis. In GC cells, IT was also found that BART20-5p prevents cell death by activating Bcl-2 associated death promoters (BAD) [18].
Some studies found that BART 2, 3, 4, 7, 8, 22 causes downregulation of caspase- 3 in NPC cell and EBV positive GC cell BART5 functions to prevent apoptosis by downregulating p53 upregulated modulator of apoptosis (PUMA). To undergo cell proliferation and to prevent apoptosis, some of the miRNAs such as BART9 causes proliferation of nasal NK/T cell lymphoma cell, and overexpression LMP1 BART10-3p is related to inhibition of apoptosis association protein. Apoptosis suppression in BLC, BART10 activate Frokhead boxP1 (FOXP1), inducing an inflammatory response in NPC and GC cell causing tumor proliferation. Hence, miRNA targets different genes, and its activation or repression leads to cell proliferation and prevention of apoptosis $[16,18]$.

\section{Cell transformation}

Cancer cells on transformation show the characteristic of growth and immortalization, whereas miRNAs responsible for cell transformation, such as miRNA BHRF1, cause cell cycle progression and inhibits apoptosis in B-cell [18]. BHRF1 in B-cell causes reduced expression of latent genes, causing persistence of the virus in cells. Some studies showed that virus presence causes cell transformation more rapidly than cells with less viral load. In EBV infected cell proliferation, the later stage of infected BHRF1 transcript elevates p27 expression. EBNA-LP expression is also modulated, which leads to B-cell transformation [16].

The EBV expressed miRNA regulates EMT activation, leading to cell transformation associated with tumor invasion, metastasis, and proliferation [8]. BART9 has been shown to play a crucial role in EMT activation by suppressing E-cadherin, causing EMT in EPV related GC, and negatively regulating miR-200a; also, targeting Ecadherin and causing invasion and migration of NPC cells. BART10$3 p$ enhances EMT markers such as snail and $\boldsymbol{\beta}$-catenin, which target BTRC gene encoding for $\boldsymbol{\beta}$-TrCP. Similarly, miRNA such as BART miRNA cluster 2, BART20-5p, causes activation of a pathway that leads to cell transformation. It was found that some miRNA such as BART6-3p causes suppression of the EMT process in NPC and GC cells [13].

BART11 increases downregulation of FOXP1, which joins the expression of E-cadherin and snail [16]. Derived tumor-associated macrophages (TAM) infected with BART11 miRNA of EBV and FOXP1 cultured along with GC cell showed EMT change later showed that BART11 reduces the expression of FOXP1 and causes 
EMT activation in GC cell causing cancer metastasis and invasion. The BART-11/FOXP1 signaling can induce NF-k $\boldsymbol{\beta}$ and IL-6, which reduces E-cadherin expression and overexpression of vimentin, $\mathrm{N}$ cadherin, and snail. These show that BART11 and BART11/FOXP1 signaling axis could be a therapeutic target for GC [19].

BART-12 is an EBV miRNA that causes cell invasion and metastasis in NPC and GC epithelial cells; it is expressed on NPC and GC tumors. Overexpressed BART-12 binds with 3'UTR of tubulin polymerization protein 1 (TPPP1), leads to reduce the expression of TPPP1, which prevents $\boldsymbol{\alpha}$-tubulin acetylation and promotes $\boldsymbol{\beta}$-catenin acetylation [8,17]. The former causes microtubule stability and lately EMT activation, whereas microtubule stability leads to the rapid division of cancer cells. In contrast, EMT activation causes invasion and metastasis of cancer cells, whereas BART12 miRNA reduces the expression of some epithelial cell markers causing transformation of NPC cells to mesenchymal [18].

\section{Action of Mechanism of EBV on Lung cancer}

More incidence of Epstein-Barr virus is found in lung cancer (LC) patients than in ordinary people; it appears that lung tissue could act as a potential storage site for the virus leading to cancer. Histologically, EBV and lung cancers are more likely to occur in Asians as a more common lymphoepithelial cancer, an uncommon type of non-small cell lung cancer (NSCLC). Several studies have shown the EBV presence in squamous cell carcinoma (LUSC) and pulmonary adenocarcinoma (LUAD) $[20,42]$.

NSCLC is related to the Epstein-Barr virus, two strains types, type I and type II: type I of EBV is more common and viral than the second type. The EBNA2, EBNA3A, EBNA3B, and EBNA3C latency genes show substantial intra-strain protection between this I and II type strains of EBV. The high EBV count has sufficient read coverage for a detailed genotype [20]. The expression of EBV genes is higher in which the BamHI-A genes are present, particularly RPMS1, A73, and the latent genes EBNA1, LMP1, LMP2A, and LMP2B are detectably expressed in contrast to EBV-low; however, latency genes of EBV type II: EBNA3A, 3B, 3C, and LP are suppressed. The poly-A region transcribed in the EBV Bam HI A genome is the superlative active region, and, in the case of LC, this ratio is more than $96.3 \%$ [5].

Another important branch is the LUAD EBV (+) probe. Studies indicate that separate molecular NSCLC subtypes with their distinct molecular markers include LUAD and LUSC. BRCA1 par- ticipates in the intrinsic sense of herpes virus DNA and the transcription of EBV. TNF receptor signaling is involved in cells with an EBV-high LUSC, which might be a shortened version of the TNF receptor due to the production of the LMP1 virus $[5,20]$.

CDKs signaling can be triggered via upright CDK5 activator P35 infections in viral expressions of EBNA-2 and EBV infection in lung cancer. With high EBV LUSC, in addition to (G2/M and G1/S cell cycle control, p53 signaling, HIPPO, and sirtuin), many cancer-associated signaling mechanisms are also activated [8]. EBV infection can suppress the overexpression of leukocytes, block the signaling of neutrophils, and activate the B-cells. Compared with LUSC EBV-low LUSC, EBV-high has more CD4 + naive T, CD8 + effector T cells, fewer CD4 cells effector $+\mathrm{T}$, and naive CD8+ T. An increased EBV infection can stimulate the invasion of immune cells into the tumors of the lung. This discovery is consistent with the idea that EBV could stimulate checkpoint molecules' production and enhance tumor egress from immune surveillance [20]. In tumor cells and transplant immune cells, their expression determines the total amount of checkpoint molecules. Essential checkpoint molecules such as IDO, PD1, CTLA-4, and VISTAs presumably significantly affect the non-small cell lung cancer expansion [21].

EBV strains cannot successfully cause expression of LAG3 and BTLA in lung cells, indicating that the significant manifestation of these molecules is in the infiltration of tumor tissue immune cells, and it seems that EBV infection might change the expression of the checkpoint molecule in cancer cells and lead to carcinogenesis of the lungs [20].

lncRNAs work in transcript repressive complexes, which increases the chance that the BamHl will act as transcripts in EBVhigh NSCLCs as IncRNAs that optionally crack cell gene expression. These BamHI A transcripts also encode at most minuscule $44 \mathrm{mi}$ croRNA BART. BART miRNAs have already been investigated for their role during the life cycle of EBV and in EBV-related malignancies [20].

BamHI's high degree of expressiveness in rightward transcripts of lung cancer may have an essential role in modulating the BART miRNA cell phenotype in lung tumors. Alternate LMP2A splicing may be more prevalent and play a vital role in the regulatory and functional role of EBV in its life cycle and pathogenesis. Despite the lack of tangible other lytic viruses' genes activation in the NSCLC- 
high EBV cases, a significant level of expression of the EBV BNLF2a gene has been identified [5,20].

The protein in the early lytic phase, namely BNLF2a, suppresses EBV immune recognition by preventing antigen presentation via inhibiting peptide load on significant molecules of the histocompatibility complex (MHC) class I. A new latency phase program results from the expression of BNLF2a, EBNA1, and LMP2 with a lack of reactivation can allow a subset of lung malignancy of EBV origin to have immunological tolerance to BNLF2a. In cases of high EBV, the BNLF2a molecule can kill the host immune system cells. The NSCLC sample with high EBV showed an increased representation of various immune checkpoint blockers such as IDO, PD1, CTLA4, LAG3, BTLA, and VISTA. Like IDO (indoleamine 2,4-dioxygenase), an immune inhibitor that is one of the primary triggers of EBV, it can block cytotoxic T-lymphocytes and NK cells by falling out of the local tumor niche tryptophan landing $[20,21]$.

\section{Action of mechanism of EBV on gastric cancer}

Globally, gastric cancer is the 5th malignant tumor with the second-highest mortality rate. EBV inserts its genes into the host genome, inducing carcinogenesis, and latently expresses microRNAs that act on methylation in the host genome leading to gastric cancer [12]. In all EBV infected cells, nuclear antigen protein 1 (EBNA1) is expressed, increasing reactive oxygen uptake by NADP oxidase responsible for malignancy. EBNA1 protein, regulated by miR34a, self-regulates NOX2 (mediated by downregulation in miRNA34a), ROS, and cell survival [22].

The results of studies on the good or bad prognosis of EpsteinBarr virus-related gastric cancer are varied. In tumors, the virus increases methylation, especially in IHH, FAM3B ACSS1, and TRABD, and significantly changes signaling pathways (conduction in the axon pathway, formation of local adhesions, interaction between cytokines and their receptors) [24]. It even changes the signal transduction of MAPK and regulates actin in the cytoskeleton affecting cell checkpoints. The virus expressed EBNA1, which promotes gastric cancer by destroying promyelocytic leukemia (PML) nuclear bodies and re-regulating NOX2 expression; it increases ROS and leads to cancer cell survival $[22,43]$.

Although the virus infects $90 \%$ of adults, EBV-related gastric cancers account for about $9 \%$ of gastric cancers and have specific pathogenic features, but their clinical signs are not specifically rec- ognizable. On average, the risk of developing this type of cancer increases with age and 2.4 times the ratio of men to women. Gastric cancer affects the cardiac and body parts of the stomach; it quickly invades the mucosa; however, it shows low lymph node metastasis and is detected more quickly in the advanced stage, and the survival time less than three years. The survival and prognosis of EBVrelated gastric cancer are better than other gastric cancers $[22,41]$.

For screening, the LMP-1 membrane protein encoded by EBV can be used. Moreover, for false-positive targets, an introductory sequence in ISH, called the EBER-1 probe, can be used to help detect the virus in gastric tumor cells with precise localization. ISH sequences: the EBV-related gastric cancer genome, anti-EBV antibodies, anti-p53, and p53 methylation are closely associated with gastric cancer, while the TP53 mutation is inversely related $[24,40]$. In addition, the virus remains latent in the body. The most fundamental step in carcinogenic gastric cancer associated with EBV (EBVaGC); is a decrease in E-cadherin expression due to the latent Epstein-Barr virus genes expression products [23].

EBNA-1 helps to bind the virus genome to the host genome and plays a vital role in latent infection and activating the virus genome, and regulating tumor activity with the help of miR-34a and NOX2, resulting in ROS accumulation [14,23].

LMP2A is the most crucial molecule that initiates gastric cancer, preventing apoptosis through the PI3K/AKT pathway, activating DNMT1 transcription, and methylation in the promoter through phosphorylation in STAT3. It also prevents AQP3 expression through ERK phosphorylation and DNMT3a transcription [5,23].

Unlike other human viruses that cannot express miRNAs, EBV encodes more than 40 types of MicroRNA; miRNA BART11 can reduce the amount of Foxp1 and increase the rate of tumor metastasis and change the survival of patients and the prognosis of the disease. In addition to BART11 and BART10-3, BART22 also affects EBV-related gastric cancer metastasis by activating the Wnt pathway. BART3-3p modulates SP to increase resistance to anticancer drugs, inhibits aging, and promotes growth. MiR-BART3$5 p$ is another MicroRNAs suppress tumors via inhibiting DICE1. EBV-miR-BART4-5p is involved in EBV-positive gastric cancer and, like EBV-miR-BART20-5p, prevents apoptosis. However, EBV-miRBART15-3p induces apoptosis by inhibiting its inhibitory genes, such as TAX1BP1 and NLRP3 $[16,18]$. 
One of the critical factors that regulate most events such as survival, proliferation, death, and metastasis is ROS. EBV promotes ROS towards metastasis; EBNA1 protein re-regulates the NOX 2 leads to ROS accumulation, whereas miR34a causes NOX2 apoptosis by regulating its gene expression in glioblastoma. There is an inverse relationship between miR34a and EBNA1 expression that studies have confirmed; by signaling miR34a-NOX2-ROS and reexpressing miR34a, EBNA1 improves cell viability [22].

Methylation is the essential mechanism associated with gastric cancer caused by the EBV, affecting 126 genes; all most methylated genes are PIK3CA and ARID1A. In addition, the amount of methylation in the promoter for the Rec8 gene is higher in EBV-related gastric cancer and may be associated with reduced patient survival. In these patients, the SSTR1 gene is expressed to suppress the tumor [24].

Excess expression of LMP1 and LMP2A suppresses COX-2 production that is regulated by a reduction in TRAF2. P-ERK helps LMP1 suppress COX-2 in gastric cancer, and LMP2A stimulated phosphorylation of STAT3 and activated DNMT1 transcribing, leading to PTEN promoter methylation on CpG island and a decrease of PTEN activation in gastric cancer associated with EBVs. Tumor cells have the potential to escape the immune system, but with the enzyme indolamine-pyrrole-2, 3-dioxygenase (ID01), it can be targeted and prevented from being rearranged. In infected tumor cells, programmed death-ligand 1 (PD-L1) increases and can be used as a prognosis factor $[5,22]$.

MiRNAs, DNA methylation, or immunotherapy treatment aims at managing this form of cancer. Recent trials have also shown that immunotherapy can assist achieve full recovery from EBV-positive stomach cancer [24].

\section{Role of IncRNA in EBV tumorigenesis}

mRNA is the transcription product of $2 \%$ of our genes. Furthermore, RNA polymerase II encodes many long non-coding RNAs (lncRNAs) from the human genome that are often 200 nucleotides long, located in both cytoplasm and nucleus of the cell, and cannot encode proteins. IncRNAs, known as the genome's dark matter and now recognized as essential regulators of cellular processes such as growth, survival, homeostasis, differentiation, migration, and genomic stability [25].
EBV encoded proteins and mRNAs can control the expression of IncRNAs in the abnormal host. EBV is implicated in $10 \%$ of gastric cancers, with lncRNAs serving as the primary regulators. NPC (Nasopharyngeal cancer) is one of the EBV-related cancers; found in nearly all low or undifferentiated NPCs. Two thousand one hundred ninety-two lncRNAs are abnormally expressed in this cancer, and 62 lncRNAs of trans-regulated genes, such as CD44, play a crucial role in cancer [26].

LINC00312 lncRNA has an anti-tumor role, preventing cell cycle progression in S to G1 phase and exacerbating apoptosis; it has an inverse relationship with EBV ncRNAs. It can be used to diagnose and treat nasopharyngeal cancer caused by EBV. The activity of EBV-miRBART6-3p overexpresses the IncRNA LOC553103 leading to proliferation, invasion, metastasis, and epithelial-mesenchymal transition (EMT) [25].

Exosomes can deliver lncRNAs into the bloodstream to communicate with cells containing proteins, mRNAs, and miRNAs. Serum levels of MALAT1, APAF1-AS1, and miR-BART 1-5p, BART 5, BART 7-3p, BART 12 and BART 13 AL359062, rise in NPC patients involved in angiogenesis, host immune system escape, division, and invasion [27].

SNHG8 expression in cancer patients helps determine the Tumor-Node-Metastasis (TNM) stage, modulating TRIM28, EIF4A2, NAP1L1 PLD3, and RPL18A in conjunction with EBV genes such as BHLF1, BHLF3, BHRF1, and BNLF2a54. In cell lysis, BHRF1 prevents apoptosis, and BNLF2a54 aids in the escape of CD8 + T cells and the immune system. Reduced SNHG8 regulation can prevent the cell cycle progression from G0 to G1 phase and facilitate apoptosis. As a result, various lncRNAs expressed in NPCs and GCs can be used as diagnostic or therapeutic targets [25,27].

MALAT1 is linked to EBV infection in the lymphoblastoid cell line, and its rearrangement promotes carcinogenesis and immune evasion in diffuse large B-cell lymphoma and the production of tolerogenic dendritic cells and regulatory $\mathrm{T}$ cells [25]. BART is highly transcribed (99\% in EBVaGC) and generates miRNAs or lncRNAs. These miRNAs accelerate the cell cycle progression, malignancy, and death [26]. BART IncRNAs control the lytic cycle of EBV replication in the cell when NF-kB is activated. As a result, they may be a therapeutic target. BART IncRNA can diminish tumor inhibitor expression by acting on the 3 ' UTR gene and modulating unfolded

Citation: Mukherjee, A.G., Wanjari, U.R., Ramezani, F., Laxmi, A., Balgote, P.J., and Dhoke, S.B. "Epstein-Barr Virus and its Capacity to Evade the Immune System to Cause Cancer". Acta Scientific Microbiology 4.9 (2021): 52-62. 
protein response (UPR) such as XBP1, ATF4, and ATF6. This UPR probably accounts for a decrease in stress-responsive genes in EBV tumor formation [25].

LMP1 is an oncoprotein expressed in NPC-infected cells and activates various biological pathways such as NF-B, JAK/STAT, and Ras/MAPK. MiR-BART16 can reduce LMP1 expression. MiRBART22 can regulate LMP2A expression, allowing viruses to evade the host immune system. MiR-BART6-5p also inhibits EBNA2, which aids in the maintenance of latency. In contrast, MiR-BART20$5 p$ inhibits BZLF1 and BRLF1 as well as cell division in the latency phase $[5,27,28]$.

MiR-BART7 promotes migration, invasion, and proliferation in NPC cells via PTEN/PI3K/Akt induction of c-Myc and c-Jun34 expression. MiR-BART7 serum levels can be used to screen NPC patients. It also reduces TGF135 protein expression and improves the effect of radiotherapy on NPCs [28].

BART1-5p kindles glycolysis, angiogenesis, and proliferation in NPC cells via the AMPK/mTOR/HIF1 pathway and causes cell migration and invasion by acting on PTEN. EBV-miR-BART-6-3p inhibits cell division to stage G1 via the LOC553103-STMN1 pathway. MiR BART1-3p acts on DAB2 to induce apoptosis and inhibit migration.

EBV-encoded miRNAs act reductively on human miRNAs, and this reductive regulation acts as an inhibitor of EBV tumorigenesis and includes the let-7 and miR-200 families. EBNA1 has a regulatory role in the expression of several miRNAs in the family, such as let-7, which aims to regulate and reduce the persistence of latent EBV infection $[22,25]$.

LncRNAs 16, 43, 54, 55 are involved in the division, migration, angiogenesis, and escape of the host immune system. EBV controls the expression of host IncRNAs and encodes lncRNAs that affect signaling pathways and proteins associated with human cells [27].

\section{Novel treatment technology}

Immunotherapy

An antiviral treatment to EBV-associated tumorigenesis lacks the immunosuppressive influence -leading cause of tumorigenesis. Passive immunization as EBV-specific cytotoxic T-lymphocyte (CTL) can provide novel treatment to the EBV-induced tumor [29].
This infusion-generated EBV-specific CTL helps prevent and eradicate lymphoproliferation recipients who had allogeneic stem cell transplants. However, autogenic and haploidentical EBV-specific CTL has also been successfully administered after human leukocyte antigen (HLA) matching. EBV-specific CTL is developed by activating peripheral blood mononuclear cells (PBMCs) from the EBV+ serotype patient [30].

Although CTL has a success rate, its production from PBMCs is intensive and effortful; therefore, another rapid process was used, which developed from tumor infiltration lymphocyte (TIL). It has a high level of T-cell specific to EBV and can be used to treat tumors. Natural killer (NK) cells use adoptive immunotherapy because NK cells can differentiate and detect viral and malignant cells [31].

\section{Antiviral}

Several antiviral drugs have gone for clinical trials and shown anti -EBV activity. Most of the drugs used in EBV have broad specificity against the herpes virus and cytomegalovirus. Generally, EBV lacks the lytic cycle; hence thymidine kinase is inhibited. Drugs like ganciclovir (a nucleoside analog) are administered with arginine butyrate in lymphoma associated with EBV; this drug requires thymidine kinase to form, a toxic metabolite causing strand termination and cell death. Omaciclovir, a nucleoside analog H2gG((R)9-[4-hydroxy-2-(hydroxymethyl) butyl] guanine), causes limited chain elongation by incorporating bases into the viral DNA as triphosphate (H2g-TP) [32].

Foscarnet act as a viral DNA polymerase, and its administration has shown to have complete suspension from EVB induced lymphoma. Cidofovir also inhibits EBV DNA polymerase; in vitro screening has shown promising nasopharyngeal lymphoma (NPC) results. Later, Cidofovir is administered along with Rituximab (an anti-CD20 mAb), which affects both oral hairy cell leukoplakia and post-transplant lymphoproliferative disorder [33].

Maribavir (MBV), a protein kinase inhibitor, has shown a high specificity against human cytomegalovirus (HCMV) disease and EBV; it was shown that MBV was responsible for inhibition of BMRF1 phosphorylation and showed specificity toward different RNA $[32,33]$.

Some thymidine derivatives such as KAY-2-41 and KAH-39-141 have shown specificity against EBV infection; they act as antivirals 
by phosphorylation of thymidine kinase (viral) cytosolic thymidine kinase1. Antivirals such as Azelaic bishydroxamic acid, an inhibitor of histone deacetylase, can kill the lymphoma cell line associated with EBV. In vitro studies revealed that zidovudine, along with IFN- $\boldsymbol{\alpha}$, causes apoptosis of cancer cells. In contrast, some chemicals such as hydroxyurea, flavanones such as amorilin and lupinifolin, and flavonoid derivatives have shown some antiviral activity $[32,34]$.

\section{Monoclonal antibody (mAb)}

Administration of targeted $\mathrm{mAb}$ gives a significant result for EBV-associated lymphoma. One such mAb is Rituximab (anti-CD20 $\mathrm{mAb}$ ), successfully treating CD20-expressing lymphomas and beneficial for managing EBV-induced lymphoproliferative disorders. Another mAb 72A1 helps to develop an Anti-gp350 antibody that functions to block EBV infection in B-cell [35].

\section{Vaccination}

Recombinant viral vector vaccines are engineered to live viruses that express some immunogenic protein to elicit an immune response; these vaccines have more advantages over other vaccines. Many viral vectors are used, such as poxvirus, adenovirus, yellow fever virus; among these, vector vaccine viruses and adenovirus elicits a good T-cell response [36]. Recombinant vaccines made against EBV: use of the vaccinia virus express BLLF1, an EBV membrane antigen, but this vaccination mode is not accepted due to its adverse effect shown in some patients. Poxvirus as a vector called modified vaccinia virus Ankara (AVA) encodes for the initial expression of six nuclear antigens (EBNA1) and two membrane proteins (LMP2) (membrane antigen), when administered, elicit an immune response in the host cell [37].

Some viral vector vaccines that elicit immune responses can neutralize and are not allowed for secondary injection. Hence, heterologous prime-boost strategies are used; in this two-antigen formula, vaccination, such, e.g., is MVA and adenovirus combination vaccine targets against EBNA1, leads to CD4+ CD8+ T-cell response and works against EBV associated lymphoma [37].

Some peptide-based vaccines are also made against EBV as EBNA1 protein fused with monoclonal antibody (mAb) and work against Dendritic cell (DC) endocytic receptor (DEC-205), enhancing the T-cell response. However, this vaccine induces CD4+ and
CD8+ response at a low level; therefore, viral vector vaccine includes CD4+ and CD8+ T-cell response [38].

\section{Conclusion}

EBV has become the day's talk, and several research studies are being undergone to find a concentrated solution and tackle it. When the immune system is suppressed, the infected cells go undetected, and the number of infected cells in the oropharynx increases, as secondary mutations, immunosuppression, and other infections stimulate proliferation [30]. The virus accumulates in the reservoir cell, and the lytic cycle repeats, after which virus burst the host cell and release more virus to infect other cells. EBV determines latent B-lymphocyte contagion by viral DNA primarily [1]. Unique programs have been developed to generate viral genes to enable infected B-cells to specialize, showing the signs of survival; necessary for B-cell stimulation and memory cell development. The study of such ideas has provided us with a piece of detailed knowledge about the whereabouts of the virus in the body. Viral Like Particle (VLP) is becoming a research tool for the EBV vaccine to design an EBV vaccine. VLP is a viral particle lacking nucleic acid; this approach is safe and can exhibit innate and adaptive immune responses. Immunogenic VLP is created using antigens such as EBNA1, EBNA3C, and ENRF1 that cause secretion of CD4+ T-cell. Such innovative strategies have provided us with new hopes to find a novel approach to handle this virus [39].

\section{Bibliography}

1. Ko YH. "EBV and human cancer". Experimental and Molecular Medicine 47.1 (2015): e130-e130.

2. Broccolo F., et al. "Human papillomavirus (HPV) and EpsteinBarr virus (EBV) in keratinizing versus non-keratinizing squamous cell carcinoma of the oropharynx". Infectious Agents and Cancer a.1 (2018): 1-5.

3. Xiao N., et al. "Regulation and evasion of host immune responses by Epstein-Barr virus". Wei sheng wu xиe bao= Acta Microbiologica Sinica 56.1 (2016): 19-25.

4. Jog NR., et al. "Epstein Barr virus nuclear antigen 1 (EBNA-1) peptides recognized by adult multiple sclerosis patient sera induce neurologic symptoms in a murine model". Journal of Autoimmunity 106 (2020): 102332. 
5. Yun SM., et al. "LMP1 and 2A Induce the Expression of Nrf2 Through Akt Signaling Pathway in Epstein-Barr Virus-Transformed B Cells". Translational Oncology 12.5 (2019): 775-783.

6. Mawson AR and Majumdar S. "Malaria, Epstein-Barr virus infection and the pathogenesis of Burkitt's lymphoma”. International Journal of Cancer 141.9 (2017): 1849-1855.

7. Gao Y., et al. "IRF4 promotes Epstein-Barr virus activation in Burkitt's lymphoma cells". Journal of General Virology 100.5 (2019): 851-862.

8. Yin H., et al. "Molecular mechanisms of EBV-driven cell cycle progression and oncogenesis". Medical Microbiology and Immunology 208.5 (2019): 573-583.

9. Montes-Mojarro IA., et al. "Epstein-Barr virus positive $\mathrm{T}$ and NK-cell lymphoproliferations: morphological features and differential diagnosis". Seminars in Diagnostic Pathology 37.1 (2020): 32-46.

10. Agarwal N., et al. "Prognostic Differences: Epstein-Barr Virus-Associated Primary Leiomyosarcoma of the Spine Versus Spinal Leiomyosarcoma Metastases". World Neurosurgery 99 (2017): 484-490.

11. Dekate J and Chetty R. "Epstein-Barr virus-associated smooth muscle tumor". Archives of Pathology and Laboratory Medicine 140.7 (2016): 718-722.

12. Qi YF., et al. "EBV down-regulates COX-2 expression via TRAF2 and ERK signal pathway in EBV-associated gastric cancer". Virus Research 272 (2019): 197735.

13. Židovec Lepej S., et al. "miRNAs: EBV mechanism for escaping host's immune response and supporting tumorigenesis". Pathogens 9.5 (2020): 353.

14. Kang BW., et al. "High level of viral microRNA-BART20-5p expression is associated with worse survival of patients with Epstein-Barr virus-associated gastric cancer". Oncotarget 8.9 (2017): 14988.

15. Sasaki S., et al. "EBV-associated gastric cancer evades T-cell immunity by PD-1/PD-L1 interactions". Gastric Cancer 22.3 (2019): 486-496.

16. Song Y., et al. "Epstein-Barr virus-encoded miR-BART11 promotes tumor-associated macrophage-induced epithelial-mesenchymal transition via targeting FOXP1 in gastric cancer". Virology 548 (2020): 6-16.
17. Wang M., et al. "The function and therapeutic potential of Epstein-Barr virus-encoded microRNAs in cancer". Molecular Therapy-Nucleic Acids 17 (2019): 657-668.

18. Wu Y., et al. "EBV-miR-BART12 accelerates migration and invasion in EBV-associated cancer cells by targeting tubulin polymerization-promoting protein 1". The FASEB Journal 34.12 (2020): 16205-16223.

19. Song Y., et al. "Epstein-Barr virus encoded miR-BART11 promotes inflammation-induced carcinogenesis by targeting FOXP1". Oncotarget 7.24 (2013): 36783.

20. Kheir F., et al. "Detection of Epstein-Barr virus infection in non-small cell lung cancer". Cancers 11.6 (2019): 759.

21. Villarroel-Espindola F., et al. "Spatially resolved and quantitative analysis of VISTA/PD-1H as a novel immunotherapy target in human non-small cell lung cancer". Clinical Cancer Research 24.7 (2018): 1562-1573.

22. Kim SM., et al. "EBV-encoded EBNA1 regulates cell viability by modulating miR34a-NOX2-ROS signaling in gastric cancer cells". Biochemical and Biophysical Research Communications 494.3-4 (2017): 550-555.

23. Shi Q., et al. "Latent membrane protein $2 \mathrm{~A}$ inhibits expression level of Smad2 through regulating miR-155-5p in EBV-associated gastric cancer cell lines". Journal of Medical Virology 92.1 (2020): 96-106.

24. Choi SJ., et al. "DNA hypermethylation induced by EpsteinBarr virus in the development of Epstein-Barr virus-associated gastric carcinoma". Archives of Pharmacal Research 40.8 (2017): 894-905.

25. Zhang J., et al. "Long noncoding RNAs involvement in EpsteinBarr virus infection and tumorigenesis". Virology Journal 17.1 (2020): 1-8.

26. Zhang J., et al. "Differential expression profiling of IncRNAs related to Epstein-Barr virus infection in the epithelial cells". Journal of Medical Virology 91.10 (2019): 1845-1855.

27. Wang H., et al. "The roles of miRNAs and lncRNAs in EpsteinBarr virus associated epithelial cell tumors". Virus Research (2020): 198217.

28. Wang L., et al. "Effects of ebv-miR-BART7 on tumorigenicity, metastasis, and TRAIL sensitivity of non-small cell lung cancer". Journal of Cellular Biochemistry 120.6 (2019): 1005710068.

Citation: Mukherjee, A.G., Wanjari, U.R., Ramezani, F., Laxmi, A., Balgote, P.J., and Dhoke, S.B. “Epstein-Barr Virus and its Capacity to Evade the Immune System to Cause Cancer". Acta Scientific Microbiology 4.9 (2021): 52-62. 
29. Lee AZE., et al. "Cellular-based immunotherapy in EpsteinBarr virus induced nasopharyngeal cancer". Oral Oncology 84 (2018): 61-70.

30. Kelly RJ. "Immunotherapy for esophageal and gastric cancer". American Society of Clinical Oncology Educational Book 37 (2017): 292-300.

31. Png YT., et al. "The Role of NK Cells in EBV Infection and EBVAssociated NPC". Viruses 13.2 (2021): 300.

32. Pagano JS., et al. "Antiviral drugs for EBV". Cancers 10.6 (2018): 197.

33. Whitehurst CB., et al. "Maribavir inhibits Epstein-Barr virus transcription through the EBV protein kinase". Journal of Virology 87.9 (2013): 5311-5315.

34. Coen N., et al. "Antiherpesvirus activities of two novel 4'-thiothymidine derivatives, KAY-2-41 and KAH-39-149, are dependent on viral and cellular thymidine kinases". Antimicrobial Agents and Chemotherapy 58.8 (2014): 4328-4340.

35. Farrell PJ. "Epstein-Barr virus and cancer". Annual Review of Pathology: Mechanisms of Disease 14 (2019): 29-53.

36. Rühl J., et al. "Vaccination against the Epstein-Barr virus". Cellular and Molecular Life Sciences (2020): 1-10.

37. Perez EM., et al. "Novel Epstein-Barr virus-like particles incorporating gH/gL-EBNA1 or gB-LMP2 induce high neutralizing antibody titers and EBV-specific T-cell responses in immunized mice". Oncotarget 8.12 (2017): 19255.

38. van Zyl DG., et al. "Immunogenic particles with a broad antigenic spectrum stimulate cytolytic T cells and offer increased protection against EBV infection ex vivo and in mice". PLoS Pathogens 14.12 (2018): e1007464.

39. Lao TD., et al. "Molecular screening for Epstein-Barr virus (EBV): detection of genomic EBNA-1, EBNA-2, LMP-1, LMP-2 among Vietnamese patients with nasopharyngeal brush samples". Asian Pacific Journal of Cancer Prevention: APJCP 18.6 (2017): 1675.

40. Mukherjee AG., et al. "The CRISPR-Cas System as a Technology to Redefine Industrial Biotechnology". Acta Scientific Microbiology 4.6 (2021): 45-52.

41. Mukherjee AG., et al. "A Review on The Usefulness of Various Eukaryotic Pigments and Metabolites in Cancer Treatment". WJPR 9.10 (2020): 587-611.
42. Mukherjee AG., et al. "A Review on the Present and Future Aspects of Various Prokaryotic Pigments and Metabolites Demonstrating Anti-Cancerous Properties". International Journal of Engineering Research and Technology 9.7 (2020): 1262-1266.

43. Ingole AA., et al. "A Review on Carcinogenic Heavy Metals". International Journal of Engineering Research and Technology 10.3 (2021): 403-411.

44. Mukherjee AG and Wanjari U. "A Short Review on Various Aspects of Gene Sequencing and Metagenomics to Study Human Gut Microbiome". International Research Journal of Engineering and Technology 7.7 (2020): 1280-1284.

\section{Volume 4 Issue 9 September 2021}

(C) All rights are reserved by Uddesh Ramesh Wanjari., et al. 